IJMMS 31:6 (2002) 329-337

PII. S0161171202105023

http://ijmms.hindawi.com

(c) Hindawi Publishing Corp.

\title{
THERMOELASTICITY WITHOUT ENERGY DISSIPATION FOR INITIALLY STRESSED BODIES
}

\author{
JUN WANG and SUSAN PALMER SLATTERY
}

Received 12 November 2001

\begin{abstract}
Thermoelastic equations without energy dissipation are formulated for a body which has previously received a large deformation and is at nonuniform temperature. A linear theory of thermoelasticity without energy dissipation for prestressed bodies is derived and the uniqueness theorem for a class of mixed initial-boundary value problems is established.
\end{abstract}

2000 Mathematics Subject Classification: 35L90, 74A15, 80A17.

1. Introduction. The so-called second sound effect has been given increasing attention in recent decades. This effect arises from the possible transport of heat by a wave propagation process rather than diffusion. Many articles have been devoted to the development of the generalized theory of thermoelasticity that predicts a finite speed for heat propagation. Lord and Shulman [11], employing a modified Fourier's law, developed what now is known as extended thermoelasticity. Green and Lindsay [6], based on an entropy production inequality proposed by Green and Laws [5], formulated temperature-rate dependent thermoelasticity that includes the temperature-rate among constitutive variables. Lebon [10] formulated heat-flux dependent thermoelasticity on the basis of a nonclassical approach to thermodynamics which includes the heat flux among the constitutive variables and assumes an equation of evolution for the heat flux. All these theories yield governing systems of hyperbolic equations and predict finite speed for heat propagation.

Recently, Green and Naghdi [7] reexamined the basic postulates of thermomechanics. They postulated three types of constitutive repose functions for the thermal phenomena and, accordingly, formulated three models of thermoelasticity. The nature of these three types of constitutive functions [8] is that when the respective theories are linearized, model I theory is the same as the classic heat conduction theory (based on Fourier's law); model II theory predicts a finite speed for heat propagation and involves no energy dissipation, now referred to as thermoelasticity without energy dissipation; model III theory permits propagation of thermal signals at both finite and infinite speeds and there is a structural difference between these field equations and those developed in [5, 6, 10, 11]. Ciarletta [3] later formulated a theory of micropolar thermoelasticity without energy dissipation. Detailed and comprehensive references to the developments of generalized thermoelasticity are found in two nice review papers by Chandrasekharaiah [1, 2].

In this paper, we adapt the postulates made by Green and Naghdi [7] and formulate a thermoelasticity theory without energy dissipation for solids which have previously 
received a large deformation and are at nonuniform temperatures. The classical theory for prestressed thermoelastic bodies has been obtained by Green [4] for the isothermal case and by Iesan [9] for the nonisothermal case.

2. Nonlinear formulae. Consider a thermoelastic solid whose configuration changes continuously, under external mechanical actions and heating, from an original reference state $\Omega_{0}$, with uniform temperature $T_{0}$ and uniform thermal-displacement $\alpha_{0}$, to a deformed state $\Omega$. Let $\partial \Omega_{0}$ and $\partial \Omega$ be the closed surface of $\Omega_{0}$ and $\Omega$, respectively. Let $X_{K}$ denote the position of a particle $X$ in the reference state, and $x_{k}=x_{k}\left(X_{1}, X_{2}, X_{3}, t\right)$ the position in the deformed state, where $t$ is the time. We assume that $x_{i}$ is sufficiently smooth and $\operatorname{det}\left(x_{i, A}\right)>0$ in order for this deformation to be possible.

In the material description, the basic nonlinear equations in thermoelasticity without energy dissipation take the form [2, 3, 8]

$$
\begin{gathered}
T_{J i, J}+\rho_{0} f_{i}=\rho_{0} \ddot{x}_{i}, \\
\rho_{0} T \dot{\eta}=\rho_{0} S+T \Phi_{K, K}
\end{gathered}
$$

in $\Omega_{0} \times\left[0, t_{0}\right)$, where $t_{0}$ is some time instant that may be infinite. In the above equations, $T$ is the absolute temperature, $S$ is the external rate of heat supply per unit mass and time, $\rho_{0}$ is the initial mass density, $T_{J i}$ is the first Piola-Kirchhoff stress tensor $\left(T_{J i} \neq T_{i J}\right), f_{i}$ is the external body force per unit mass, $\eta$ is the entropy per unit mass, $\Phi_{K}$ is the internal entropy flux vector. If $Q_{K}$ denotes the flux of heat across surfaces in the deformed body that were originally coordinate planes perpendicular to the $X_{K}$-axes through the point $X$, measured per unit time, then $Q_{K}=T \Phi_{K}$. Through this paper, a superposed dot stands for the material time derivative while a comma followed by a subscript denotes partial derivatives with respect to the spatial coordinates, in the deformed configuration if the subscript is a minuscule, in the reference configuration if the subscript is a majuscule; that is, $g_{, J}=\partial g / \partial X_{J}$ and $g_{, i}=\partial g / \partial x_{i}$. Einstein summation on repeated indices is also used throughout this paper.

The constitutive variables are the strain tensor $E_{A B}, T$, and $\alpha_{J}$ with the geometric equations

$$
2 E_{A B}=x_{i, A} x_{i, B}-\delta_{A B},
$$

where $\alpha$ is the thermal-displacement ( $w \dot{\alpha}=T$ ) and $\delta_{A B}$ is the Kronecker delta.

The constitutive equations are

$$
\begin{gathered}
\Psi=\Psi\left(E_{I J}, T, \alpha_{, J}\right), \\
T_{J I}=\frac{\partial \Psi}{\partial E_{J I}}, \quad T_{J i}=x_{i, I} T_{J I}, \\
\rho_{0} \eta=-\frac{\partial \Psi}{\partial T}, \quad \Phi_{K}=\frac{\partial \Psi}{\partial \alpha_{, K}},
\end{gathered}
$$

where $\Psi$ is the Helmholtz free-energy per unit volume.

Unlike other theories of hyperbolic thermoelasticity, in obtaining the above constitutive equations, the second law of thermodynamics in the form of an entropy production inequality was not used. Rather, this inequality is automatically satisfied [2, 8]. 
3. Small deformation superposed on a large deformation. In this section, we consider three states of the solid, the initial state $\Omega_{0}$, the first deformed state $\Omega$, and the final deformed state $\Omega^{*}$ corresponding respectively to the uniform temperature $T_{0}$, uniform thermal-displacement $\alpha_{0}$ and zero displacement; temperature $T$, thermaldisplacement $\alpha$, and displacement $v_{i}$; and temperature $T^{*}$, thermal displacement $\alpha^{*}$ and displacement $v_{i}+u_{i}$. Following Iesan [9], we call $\Omega$ the primary state and $\Omega^{*}$ the secondary state. Thermodynamic quantities and forces associated with $\Omega^{*}$ are denoted by an asterisk. The position coordinates of the particle $X$ at time $t$ in $\Omega^{*}$ will be denoted by $y_{i}=y_{i}\left(X_{1}, X_{2}, X_{3}, t\right)$ with $\operatorname{det}\left(y_{i, A}\right)>0$.

We define the incremental displacement $u_{i}$ and incremental temperature $\theta$ as

$$
u_{i}=y_{i}-x_{i}, \quad \theta=T^{*}-T
$$

Here we consider the case in which $u_{i}$ and $\theta$ are small; that is, there exists a real parameter $\varepsilon$, small enough for its square and higher powers to be neglected, such that

$$
u_{i}=\varepsilon u_{i}^{\prime}, \quad \theta=\varepsilon \theta^{\prime},
$$

where $u_{i}^{\prime}$ and $\theta^{\prime}$ are independent of $\varepsilon$.

In the secondary state $\Omega^{*}$, the basic equations are of the same form as those in the primary state $\Omega$. We have the equation of motion

$$
T_{J i, J}^{*}+\rho_{0} f_{i}^{*}=\rho_{0} \ddot{y}_{i},
$$

the energy equation

$$
\rho_{0} T^{*} \dot{\eta}^{*}=\rho_{0} S^{*}+T^{*} \Phi_{K, K}^{*},
$$

the geometric equations

$$
2 E_{A B}^{*}=y_{i, A} y_{i, B}-\delta_{A B},
$$

and the constitutive equations

$$
\begin{gathered}
\Psi^{*}=\Psi\left(E_{I J}^{*}, T^{*}, \alpha_{, K}^{*}\right), \\
T_{J I}^{*}=\frac{\partial \Psi^{*}}{\partial E_{J I}^{*}}, \quad T_{J i}^{*}=y_{i, I} T_{J I}^{*}, \\
\rho_{0} \eta^{*}=-\frac{\partial \Psi^{*}}{\partial T^{*}}, \quad \Phi_{K}^{*}=\frac{\partial \Psi^{*}}{\partial \alpha_{, K}^{*}} .
\end{gathered}
$$

Next, we derive the equations satisfied by $u_{i}$ and $\theta$. Keeping (3.2) in mind, we get the following second-order approximation:

$$
\begin{aligned}
\Psi\left(E_{I J}^{*}, T^{*}, \alpha_{, K}^{*}\right)-\Psi\left(E_{I J}, T, \alpha_{, K}\right)= & a_{I J}\left(E_{I J}^{*}-E_{I J}\right)+a_{K}\left(\alpha_{, K}^{*}-\alpha_{, K}\right)-a \theta \\
& +\frac{1}{2} C_{I J M N}\left(E_{I J}^{*}-E_{I J}\right)\left(E_{M N}^{*}-E_{M N}\right)-b_{I J}\left(E_{I J}^{*}-E_{I J}\right) \theta \\
& +\frac{1}{2} b_{I J K}\left(E_{I J}^{*}-E_{I J}\right)\left(\alpha_{, K}^{*}-\alpha_{, K}\right)+\frac{1}{2} d \theta^{2} \\
& -d_{K}\left(\alpha_{, K}^{*}-\alpha_{, K}\right) \theta+\frac{1}{2} d_{K L}\left(\alpha_{, K}^{*}-\alpha_{, K}\right)\left(\alpha_{, L}^{*}-\alpha_{, L}\right),
\end{aligned}
$$


where $b_{I J}, b_{I J K}$, and $d_{I J}$ have certain symmetries in their indices, and that

$$
\begin{gathered}
C_{I J M N}=C_{M N I J}=C_{J I M N}, \quad a_{K}=\frac{\partial \Psi}{\partial \alpha_{, K}}=\Phi_{K}, \\
a=-\frac{\partial \Psi}{\partial T}=\rho_{0} \eta, \quad a_{I J}=\frac{\partial \Psi}{\partial E_{I J}}=T_{I J} .
\end{gathered}
$$

Substituting (3.9) into (3.7) and (3.8), we obtain

$$
\begin{aligned}
\rho_{0} \eta^{*} & =\rho_{0} \eta+d \theta+b_{I J}\left(E_{I J}^{*}-E_{I J}\right)+d_{K}\left(\alpha_{, K}^{*}-\alpha_{, K}\right), \\
T_{I J}^{*} & =T_{I J}+C_{I J M N}\left(E_{M N}^{*}-E_{M N}\right)-b_{I J} \theta+b_{I J K}\left(\alpha_{, K}^{*}-\alpha_{, K}\right), \\
\Phi_{K}^{*} & =\Phi_{K}+b_{I J K}\left(E_{I J}^{*}-E_{I J}\right)-d_{K} \theta+d_{K L}\left(\alpha_{, L}^{*}-\alpha_{, L}\right) .
\end{aligned}
$$

Since it is required that $\Phi_{K}$ vanishes at equilibrium, that is, for $\alpha_{, K}=0$ and $T_{, K}=0$, we also must have that

$$
b_{I J K}=0, \quad d_{K}=0 \quad \text { if } T_{, K}=0 .
$$

From geometric equations and the relation

$$
y_{i, A}=x_{i, A}+u_{i, A}
$$

we get that

$$
2 E_{A B}^{*}=2 E_{A B}+x_{i, A} u_{i, B}+x_{i, B} u_{i, A},
$$

where we have used the fact that $u_{i} s$ are small, and hence, the terms like $u_{i, A} u_{i, B}$ have been neglected. Introducing the notation

$$
e_{A B}=\frac{1}{2}\left(x_{i, A} u_{i, B}+x_{i, B} u_{i, A}\right)
$$

we find that

$$
E_{A B}^{*}=E_{A B}+e_{A B}
$$

Let

$$
\tau=\int_{0}^{t}\left(T^{*}-T\right) d t=\int_{0}^{t} \theta d t
$$

we have

$$
\begin{aligned}
& \alpha=\int_{0}^{t}\left(T-T_{0}\right) d t+T_{0} t+\alpha_{0}, \quad \alpha^{*}=\int_{0}^{t}\left(T^{*}-T_{0}\right) d t+T_{0} t+\alpha_{0}, \\
& \alpha^{*}-\alpha=\int_{0}^{t}\left(T^{*}-T_{0}\right) d t-\int_{0}^{t}\left(T-T_{0}\right) d t=\tau, \quad \alpha_{, K}^{*}-\alpha_{, K}=\tau_{, K} .
\end{aligned}
$$

Substituting (3.17) and (3.20) into (3.12), we find that

$$
\begin{aligned}
\rho_{0} \eta^{*} & =\rho_{0} \eta+d \theta+b_{I J} e_{I J}+d_{K} \tau_{, K}, \\
T_{I J}^{*} & =T_{I J}+C_{I J M N} e_{M N}-b_{I J} \theta+b_{I J K} \tau_{, K}, \\
\Phi_{K}^{*} & =\Phi_{K}+b_{I J K} e_{I J}-d_{K} \theta+d_{K L} \tau_{, L} .
\end{aligned}
$$


From (2.5), (3.7), (3.14), and (3.22), we find that

$$
T_{J i}^{*}=T_{J i}+T_{J I} u_{i, I}+C_{J I M N} x_{i, I} e_{K L}-b_{J I} x_{i, I} \theta+b_{J I K} x_{i, I} \tau_{, K} .
$$

If we denote

$$
\pi_{J i}=T_{J i}^{*}-T_{J i}, \quad \varphi_{K}=\Phi_{K}^{*}-\Phi_{K}, \quad \gamma=\eta^{*}-\eta,
$$

then we have

$$
\begin{aligned}
\rho_{0} \gamma & =d \theta+b_{I J} e_{I J}+d_{K} \tau_{, K}, \\
\pi_{J i} & =T_{J I} u_{i, I}+C_{J I M N} x_{i, I} e_{M N}-b_{J I} x_{i, I} \theta+b_{J I K} x_{i, I} \tau_{, K}, \\
\varphi_{K} & =b_{I J K} e_{I J}-d_{K} \theta+d_{K L} \tau_{, L} .
\end{aligned}
$$

Subtracting (2.1) and (2.2) from (3.3) and (3.4), respectively, we find the incremental equations

$$
\pi_{J i, J}+\rho_{0} F_{i}=\rho_{0} \ddot{u}_{i}, \quad \rho_{0} T \dot{\gamma}=\rho_{0} s+\varphi_{K, K},
$$

where $F_{i}=f_{i}^{*}-f_{i}$ and $s=S^{*}-S$.

4. Linear theory of thermoelasticity for initially stressed bodies. In this section, we consider the special case when the primary state $\Omega$ of the solid is identical with that of the initial solid $\Omega_{0}$ so that $x_{1}=X_{1}, x_{2}=X_{2}, x_{3}=X_{3}$, and we suppose that $\Omega_{0}$ is subjected to an initial stress and an initial heat flux caused by the nonuniform initial temperature $T_{0}$. Due to the action of external loadings and heating, the solid $\Omega_{0}$ undergoes a deformation. There arise displacements $u_{i}=\epsilon u_{i}^{\prime}$, thermal displacement $\alpha$, and temperature increment $\theta=\epsilon \theta^{\prime}$. Here, we systematically neglect all powers of $\epsilon$ above the first, except in the free energy function $\Psi$, where we retain quadratic terms of $\epsilon$.

The work of Section 3 can be applied to this special case and yields a linear theory of thermoelasticity without energy dissipation in the presence of initial stress and heat flux. In this case, we have

$$
x_{i, I}=\delta_{i I}, \quad T=T_{0}, \quad E_{A B}=0, \quad \alpha=\alpha_{0}, \quad \dot{\tau}=\theta .
$$

All coefficients defined in the previous section are now evaluated at $E_{A B}=0, \alpha=\alpha_{0}$, and $T=T_{0}$. For convenience, from now on, we change all the majuscule subscripts into minuscule subscripts. From (3.17), we find that

$$
e_{i j}=\frac{1}{2}\left(u_{i, j}+u_{j, i}\right) \text {. }
$$

The governing equations (3.27) become

$$
\pi_{j i, j}+\rho_{0} F_{i}=\rho_{0} \ddot{u}_{i}, \quad \rho_{0} T_{0} \dot{\gamma}=\rho_{0} s+T_{0} \varphi_{k, k} .
$$

The constitutive relations (3.26) reduce to

$$
\begin{aligned}
\rho_{0} \gamma & =d \theta+b_{i j} u_{i, j}+d_{K} \tau_{, k}, \\
\pi_{i j} & =d_{i j m n} u_{m, n}-b_{i j} \theta+b_{i j k} \tau_{, k}, \\
\varphi_{k} & =b_{i j k} u_{i, j}-d_{k} \theta+d_{k m} \tau_{, m},
\end{aligned}
$$

where $d_{i j m n}=C_{i j m n}+\delta_{j m} T_{i n}$ with $\delta_{j m}$ being the usual Kronecker delta. 
The coefficients $C_{i j m n}, b_{i j}, b_{i j k}, d_{k m}, d_{k}$, and $d$ are functions of the position coordinates. The coefficients $C_{i j m n}$ have the symmetry as in (3.10), $b_{i j}$ and $d_{k m}$ have the usual symmetry about their indices and that $b_{i j k}=b_{j i k}$.

The functions $T_{\text {in }}$ characterize the initial stress and they can be arbitrary functions apart from satisfying the energy equation for the static case and the condition that $\Omega_{0}$ is in equilibrium. The presence of the initial heat flux vector is determined by the nonuniformity of the initial temperature $T_{0}$. This fact implies the appearance of the coefficients $b_{i j k}$ and $d_{k}$ in the expression for $\varphi_{k}$. If $T_{0}$ is constant, then $b_{i j k}=0$ and $d_{k}=0$.

Substituting (4.4) into (4.3), we find the governing equations in the linear theory of thermoelasticity for initially stressed bodies

$$
\begin{gathered}
\left(d_{j i m n} u_{m, n}-b_{i j} \theta+b_{i j k} \tau_{, k}\right)_{, j}+\rho_{0} F_{i}=\rho_{0} \ddot{u}_{i}, \\
T_{0}\left(d \ddot{\theta}+b_{i j} \ddot{u}_{i, j}+d_{k} \dot{\theta}_{, k}\right)-T_{0}\left(b_{i j k} \dot{u}_{i, j}-d_{k} \dot{\theta}+d_{k m} \theta_{, m}\right)_{, k}=\rho_{0} \dot{s} .
\end{gathered}
$$

If $T_{\text {in }}=0$ and $T_{0}$ is a constant, for nonhomogeneous and anisotropic materials, the above equations reduce to

$$
\begin{gathered}
\left(C_{i j m n} u_{m, n}-b_{i j} \theta\right)_{, j}+\rho_{0} F_{i}=\rho_{0} \ddot{u}_{i}, \\
T_{0}\left(d \ddot{\theta}+b_{i j} \ddot{u}_{i, j}\right)-T_{0}\left(d_{k m} \theta_{, m}\right)_{, k}=\rho_{0} \dot{s} .
\end{gathered}
$$

For homogeneous and isotropic materials, the above equations reduce to the same set of governing equations as developed in [8].

5. Uniqueness theorem. In this section, we establish a uniqueness theorem for a class of initial-boundary value problems associated with the governing equations (4.5) and (4.6) for prestressed solids.

In the context of the theory considered, an initial-boundary value problem consists in determining the functions $u_{i}(x, t)$ and $\theta(x, t)$ for $x \in \Omega$ and $t>0$, by solving (4.5) and (4.6) with $F_{i}(x, t), s(x, t), T_{i n}$, and $T_{0}$ being prescribed functions for $x \in \Omega$ and $t>0$; under the initial conditions

$$
u_{i}(x, 0)=0, \quad \dot{u}_{i}(x, 0)=0, \quad \theta(x, 0)=0, \quad \dot{\theta}(x, 0)=0 \quad \text { for } x \in \Omega,
$$

and the boundary conditions for $t>0$

$$
\begin{array}{cccc}
u_{i}=\bar{u}_{i} & \text { for } x \in \partial \Omega_{1}, & \pi_{j i} n_{j}=\bar{p}_{i} & \text { for } x \in \partial \Omega_{1}^{c}, \\
\theta=\bar{\theta} & \text { for } x \in \partial \Omega_{2}, & T_{0} \varphi_{k} n_{k}=\bar{q} & \text { for } x \in \partial \Omega_{2}^{c},
\end{array}
$$

where $\bar{u}_{i}, \bar{p}_{i}, \bar{\theta}$, and $\bar{q}$ are prescribed functions in the domains of their definition, $\partial \Omega_{i}+\partial \Omega_{i}^{c}=\partial \Omega$ for $i=1,2$ and $n_{i}$ is the outward normal vector to $\partial \Omega$.

To establish the uniqueness theorem, we impose the following restrictions on the material constants:

(i) the initial mass density $\rho_{0}$ and the specific heat $d$ are strictly positive; that is, there exist positive constants $r$ and $d_{0}$ with the same dimensions as of $\rho_{0}$ and $d$, respectively, such that

$$
\rho_{0} \geq r>0, \quad d \geq d_{0}>0
$$


(ii) $d_{i j m n}$ is positive definite in the sense that there exists a positive constant $\beta$ with the same dimension of $d_{i j m n}$ such that

$$
d_{i j m n} \xi_{i j} \xi_{m n} \geq \beta \xi_{i j} \xi_{i j}, \quad d_{i j m n}=d_{m n i j}=d_{j i m n}
$$

for all second order tensors $\xi_{i j}$;

(iii) the heat conductivity tensor $d_{k m}$ is symmetric and positive definite in the sense that there exists a positive constant $b$ such that

$$
d_{k m} \xi_{k} \xi_{m} \geq b \xi_{k} \xi_{k}, \quad d_{k m}=d_{m k}
$$

for all vectors $\xi_{k}$;

(iv) there exists a constant $h$ with the same dimension of $b$ such that

$$
4 b_{i j k}^{2}<\beta h, \quad h<b .
$$

THEOREM 5.1. If assumptions (i), (ii), (iii), and (iv) hold and $T_{0}>0$, then there exists at most one solution to the initial-boundary value problem defined by (4.5), (4.6), (5.1), and (5.2).

Proof. It suffices to show that for $F_{i}=0, \dot{s}=0$, and homogeneous boundary conditions, the solution is trivial.

From (4.5), the homogeneous boundary conditions and integration by parts, we get

$$
\begin{aligned}
\int_{\Omega} \rho_{0} \ddot{u}_{i} \dot{u}_{i} d \Omega & =\int_{\Omega}\left(d_{i j m n} u_{m, n}-b_{i j} \theta+b_{i j k} \tau_{, k}\right)_{, j} \dot{u}_{i} d \Omega \\
& =-\int_{\Omega}\left(d_{i j m n} u_{m, n}-b_{i j} \theta+b_{i j k} \tau_{, k}\right) \dot{u}_{i, j} d \Omega ;
\end{aligned}
$$

that is,

$$
\int_{\Omega}\left(\rho_{0} \ddot{u}_{i} \dot{u}_{i}+d_{i j m n} \dot{u}_{i, j} u_{m, n}-b_{i j} \dot{u}_{i, j} \theta+b_{i j k} \tau_{, k} \dot{u}_{i, j}\right) d \Omega=0 .
$$

Taking into consideration the homogeneous initial conditions and integrating (4.6), we find

$$
\left(d \dot{\theta}+b_{i j} \dot{u}_{i, j}+d_{k} \theta_{, k}\right)-\left(b_{i j k} u_{i, j}-d_{k} \theta+d_{k m} \boldsymbol{\tau}_{, m}\right)_{, k}=0 .
$$

Multiplying this by $\theta$ and integrating over $\Omega$, we find

$$
\int_{\Omega}\left(d \dot{\theta}+b_{i j} \dot{u}_{i, j}+d_{k} \theta_{, k}\right) \theta d \Omega-\int_{\Omega}\left(b_{i j k} u_{i, j}-d_{k} \theta+d_{k m} \tau_{, m}\right)_{, k} \theta d \Omega=0
$$


Using the homogeneous boundary conditions and integration by parts, we find

$$
\int_{\Omega}\left[d \dot{\theta} \theta+d_{k m} \theta_{, k} \boldsymbol{T}, m+b_{i j k} u_{i, j} \theta_{, k}+b_{i j} \theta \dot{u}_{i, j}\right] d \Omega=0 .
$$

Adding (5.8) and (5.11), we find

$$
\int_{\Omega}\left[\rho_{0} \ddot{u}_{i} \dot{u}_{i}+d_{i j m n} \dot{u}_{i, j} u_{m, n}+d \dot{\theta} \theta+d_{k m} \theta_{, k} \tau_{, m}+b_{i j k} \frac{d}{d t}\left(u_{i, j} \tau_{, k}\right)\right] d \Omega=0
$$

that is,

$$
\frac{d}{d t} \int_{\Omega}\left[\rho_{0} \dot{u}_{i} \dot{u}_{i}+d_{i j m n} u_{i, j} u_{m, n}+d \theta^{2}+d_{k m} \tau_{, k} \tau_{, m}+2 b_{i j k} u_{i, j} \tau_{, k}\right] d \Omega=0 .
$$

Taking into consideration the homogeneous initial conditions, we find

$$
\int_{\Omega}\left[\rho_{0} \dot{u}_{i} \dot{u}_{i}+d_{i j m n} u_{i, j} u_{m, n}+d \theta^{2}+d_{k m} \tau_{, k} \tau_{, m}+2 b_{i j k} u_{i, j} \tau_{, k}\right] d \Omega=0 .
$$

Using Schwartz inequality, we find

$$
\left|2 b_{i j k} u_{i, j} \tau_{, k}\right| \leq \frac{\beta}{2} u_{i, j} u_{i, j}+\frac{4 b_{i j k}^{2}}{\beta} \tau_{, k} \tau_{, k} \leq \frac{\beta}{2} u_{i, j} u_{i, j}+h \tau_{, k} \tau_{, k} .
$$

From (5.14), (5.15), and the assumption (i), (ii), (iii), and (iv), we find that

$$
\int_{\Omega}\left[r \dot{u}_{i} \dot{u}_{i}+\frac{\beta}{2} u_{i, j} u_{i, j}+d_{0} \theta^{2}+(b-h) \tau_{, k} \tau_{, k}\right] d \Omega \leq 0 .
$$

Equation (5.16) readily yields the trivial solution

$$
u_{i}(x, t)=0, \quad \theta(x, t)=0, \quad \text { for } x \in \Omega, t \geq 0 .
$$

\section{REFERENCES}

[1] D. S. Chandrasekharaiah, Thermoelasticity with second sound: a review, Appl. Mech. Rev. 39 (1986), 355-376.

[2] _ _ Hyperbolic thermoelasticity: a review of recent literature, Appl. Mech. Rev. 51 (1998), 705-729.

[3] M. Ciarletta, A theory of micropolar thermoelasticity without energy dissipation, J. Thermal Stresses 22 (1999), no. 6, 581-594.

[4] A. E. Green, Thermoelastic stresses in initially stressed bodies, Proc. Roy. Soc. Ser. A 266 (1962), 1-19.

[5] A. E. Green and N. Laws, On the entropy production inequality, Arch. Rational Mech. Anal. 45 (1972), no. 1, 47-53.

[6] A. E. Green and K. A. Lindsay, Thermoelasticity, J. Elasticity 2 (1972), 1-7.

[7] A. E. Green and P. M. Naghdi, A re-examination of the basic postulates of thermomechanics, Proc. Roy. Soc. London Ser. A 432 (1991), no. 1885, 171-194.

[8] _ Thermoelasticity without energy dissipation, J. Elasticity 31 (1993), no. 3, 189208.

[9] D. Iesan, Incremental equations in thermoelasticity, J. Thermal Stresses 3 (1980), 41-56.

[10] G. Lebon, A generalized theory of thermoelasticity, J. Tech. Phys. 23 (1982), 37-46. 
[11] H. Lord and Y. Shulman, A generalized dynamical theory of thermoelasticity, J. Mech. Phys. Solids 15 (1967), 299-309.

Jun Wang: Department of Mathematics and Computer Science, Alabama State UNIVERSITY, MONTGOMERY, AL 36104, USA

E-mail address: junwang@asunet.a1asu.edu

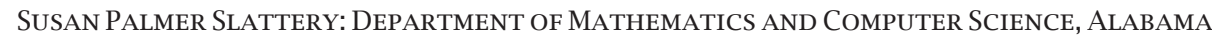
StATE UniVersity, MONTGOMery, AL 36104, USA

E-mail address: ss 1attery@asunet. a1asu.edu 


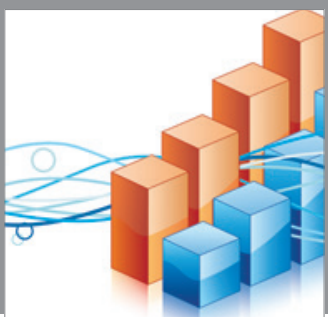

Advances in

Operations Research

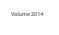

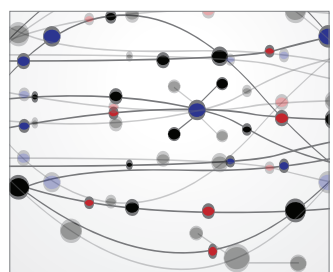

\section{The Scientific} World Journal
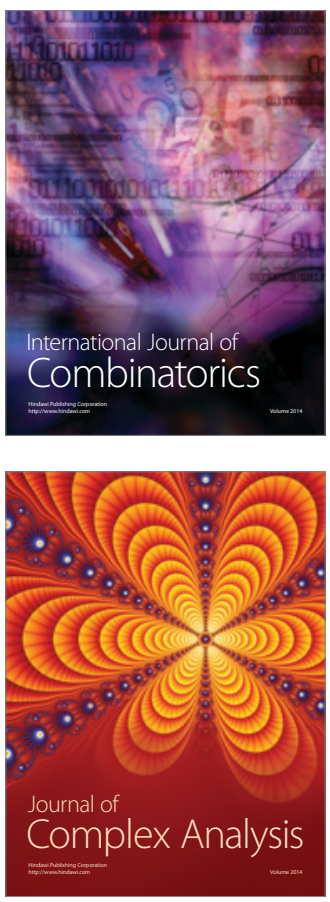

International Journal of

Mathematics and

Mathematical

Sciences
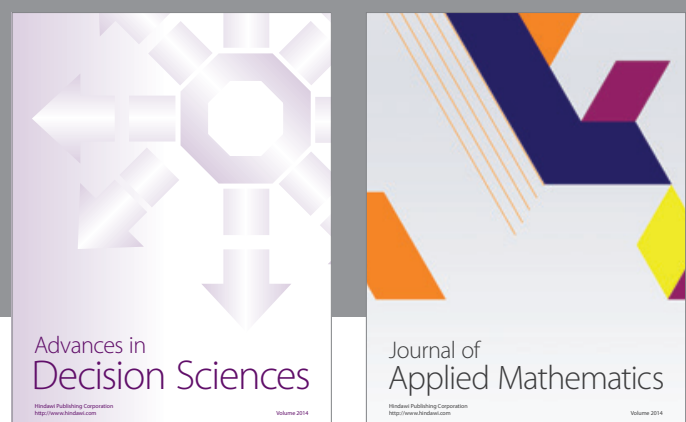

Journal of

Applied Mathematics
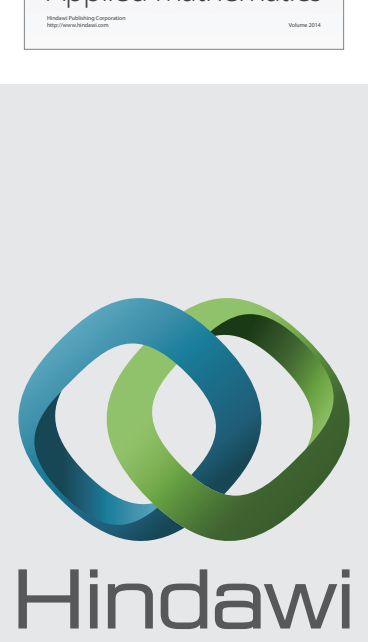

Submit your manuscripts at http://www.hindawi.com
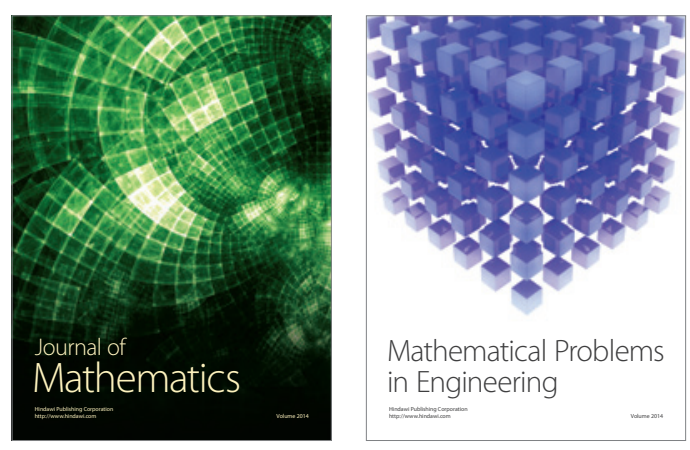

Mathematical Problems in Engineering
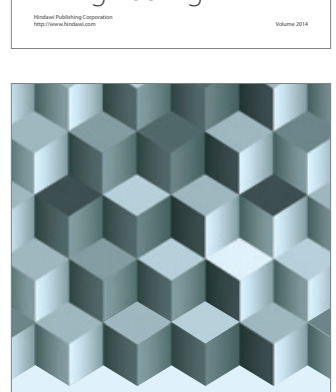

Journal of

Function Spaces
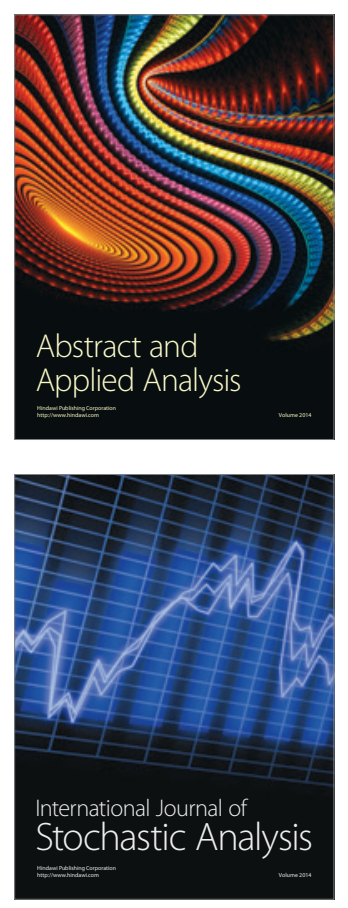

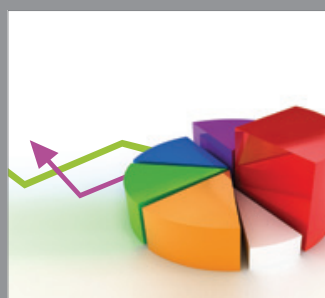

ournal of

Probability and Statistics

Promensencen
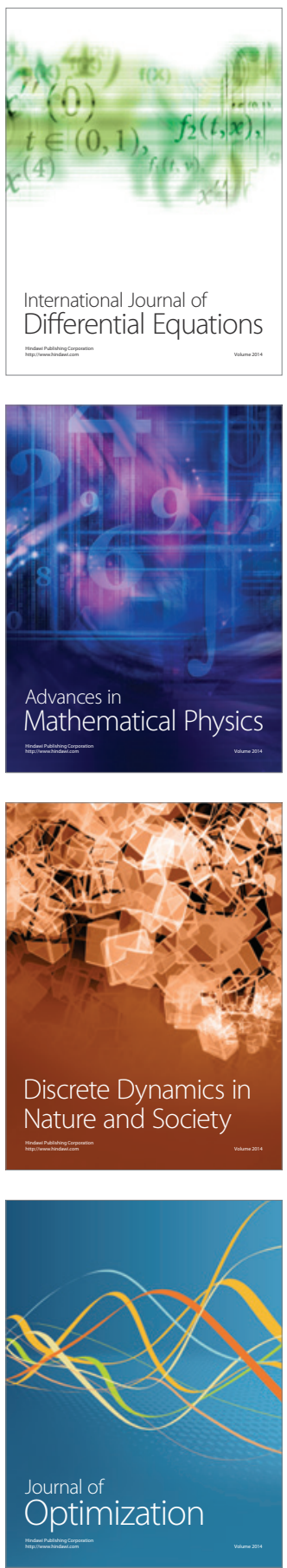